\title{
Análisis discursivo de la violencia filioparental en diferentes producciones audiovisuales
}

\author{
Laura Domínguez de la Rosa ${ }^{1}$;nmaculada Palma García²; María de las Olas Palma
}

Recibido: 23 de julio de 2019 / Revisado: 17 de septiembre de 2019 / Aceptado: 11 de noviembre de 2019

Resumen. Este trabajo pretende conocer el discurso desde el que se construye el fenómeno de la violencia filioparental (VFP). Se recurre al análisis de producciones audiovisuales que presentan esta realidad. El método utilizado es cualitativo, concretamente el análisis del discurso desde la propuesta de Potter y Wheterell de los repertorios interpretativos. Se identifican cuatro repertorios: "Construyendo el perfil de la violencia", "Amparo del silencio", "Patología del amor" y "Un síntoma de responsabilidad”. Se comprobó que a través de estos medios se contribuye a una doble visibilización de la violencia filioparental al destapar las experiencias y sentimientos presentes en las familias que viven esta situación, y a su vez, reclamar el compromiso y responsabilidad de todas las esferas sociales implicadas en su prevención y atención.

Palabras clave: violencia filioparental; análisis del discurso; producciones audiovisuales; repertorio interpretativo; método cualitativo.

\section{[en] Discursive analysis of child-to-parent violence in various media productions}

\begin{abstract}
The aim of this paper is to identify the discourse that acts as a basis for construction of the phenomenon of child-to-parent violence. Media productions that present this reality are analysed using a qualitative method, specifically the discourse analysis from Potter and Wetherell's proposal regarding interpretative repertoires. Four repertoires are identified: "Constructing the profile of violence"; the "Refuge of silence"; the "Pathology of love" and "A symptom of responsibility". We observe that these means contribute to a dual visibilisation of child-to-parent violence in terms of both uncovering the experiences and feelings of families experiencing this situation and also demanding commitment and responsibility from all social spheres involved in child-to-parent violence prevention and care.
\end{abstract}

Keywords: child-to-parent violence; discourse analysis; media productions; interpretative repertoire; qualitative method.

Sumario: Introducción. 1. Material y método. 1.1. Materiales 1.2 Método. 1.3. Procedimiento de análisis. 2. Resultados. 2.1 Construyendo el perfil de la violencia. 2.2 Amparo del silencio. 2.3 Patología del amor. 2.4 Un síntoma de responsabilidad. 3. Discusión y conclusiones. 4. Referencias bibliográficas.

Cómo citar: Domínguez de la Rosa, L.; Palma García, I.; de las Olas Palma, M. (2020) Análisis discursivo de la violencia filioparental en diferentes producciones audiovisuales, en Cuad. trab. soc. 33(1), 115-126.

Universidad de Málaga, España

1dominguez@uma.es

$2 \quad$ Servicio Integral de Medio Abierto - Fundación Diagrama

ipalma@diagrama.org

3 Universidad de Málaga, España

mpalma@uma.es 


\section{Introducción}

La violencia se ha producido siempre a lo largo de la historia por distintos motivos y de diferentes formas. En la actualidad han surgido nuevas expresiones de la misma tanto en el ámbito escolar (bullying o acoso), como en el trabajo (mobbing) o en la familia (violencia de género o intergeneracional) (Gómez et al., 2007).

En esta última realidad, en la familia, se han producido importantes cambios que afectan a sus funciones, sus roles e incluso su composición. Se ha pasado de un modelo familiar único a un escenario complejo de múltiples modelos familiares, tales como: familias monoparentales, reconstituidas, adoptantes, de acogida, etc., en los que conviven, a su vez, nuevas formas de violencia intrafamiliar (Domínguez y Montalbán, 2017). A pesar de estos cambios socioculturales y en los roles tradicionales, la institución familiar sigue siendo la primera estancia de socialización y educación en la infancia y adolescencia (Ibabe, 2015).

Sin embargo, para autores como Alba (2006) la familia es la institución más violenta de nuestra sociedad. En esta línea, se considera un grupo social complejo y de difícil organización. Por ello, la institución familiar puede ser un espacio afectivo de convivencia, de protección y de satisfacción de las necesidades o, por el contrario, puede convertirse en una fuente de conflictos y riesgos para cualquier tipo de maltrato (Alba, 2006; Aroca, Cánovas y Alba, 2012).

En la actualidad, investigaciones sobre el maltrato infantil y el maltrato a personas mayores siguen constatando la presencia de comportamientos violentos hacia los sujetos familiares más vulnerables, aun cuando muchos de estos permanecen invisibilizados (Castilla y Palma, 2014; Molina y Moreno, 2015; Peligero, 2016). Toda esta realidad de violencia familiar incluye experiencias relacionadas con la violencia de género (Medina, Arévalo y Durán, 2015), hacia personas mayores, hacia menores, entre hermanos y violencia de menores hacia ascendentes o violencia filioparental (VFP), fenómeno en el que se centra el presente estudio.

La violencia filioparental se conceptualiza, por primera vez, en el año 1957 como "síndrome de padres maltratados" (Sears, Maccoby y Levin, 1957). Anteriormente, estos hechos se explicaban como defensa de los adolescentes y jóvenes ante agresiones, abusos sexuales o trato vejatorio. También, se consideraban como forma de protección de otro miembro de la familia que pudiera ser agredido; o como devolución del maltrato o abuso que sufrieran los hijos por parte de sus padres. Este fenómeno era conocido como violencia filioparental tradicional, mientras que en los últimos años su dimensión ha alcanzado mayores cotas de presencia en familias aparentemente "normalizadas" que pertenecen a todas las clases sociales y que previamente no contaban con ninguna problemática a resaltar.

La violencia filioparental es un fenómeno cuya descripción aún no está clara y por eso es complicado conceptualizar todos los factores que se ven involucrados en este tipo de actos violentos (Calvete, Orue y González-Cabrera, 2017). No obstante, una de las definiciones que contempla una gran cantidad de elementos es la propuesta por Aroca, Lorenzo y Miró (2014). Estos autores defienden que la violencia filioparental es aquella en la que los hijos actúan consciente e intencionalmente de forma reiterada en el tiempo, con el deseo de causar daño, perjuicio y/o sufrimiento a sus progenitores, con el fin de obtener poder, control y dominio sobre sus víctimas para conseguir lo que desean.

Se produce como resultado de un ciclo de coerciones entre agresor y víctima que comienza con una actitud sumisa por parte de los padres ante la conducta violenta de sus hijos. Este ciclo continúa con una mayor exigencia de los hijos y, por lo tanto, una mayor frustración de sus padres. En general, este hecho finaliza con nueva reacción de venganza y agresividad de los hijos hacia los padres, quienes muestran de nuevo una conducta sumisa. De acuerdo con Omer (2004), una vez que se inicia el ciclo de la violencia, los hijos orientan cada vez más este acto hacia el poder mientras que los padres lo dirigen hacia la indefensión. En consecuencia, la violencia filioparental tradicional comienza a contemplarse como un fenómeno emergente o como un nuevo modelo de violencia filioparental (Pereira, 2006; Vázquez, Romo, Rojas, González y Yedra, 2019).

Así, la investigación sobre violencia filioparental se ha aproximado a este fenómeno desde diferentes teorías. Por un lado, la Teoría del aprendizaje social (Bandura, 1971) explica la conducta violenta de los hijos hacia sus padres como conductas aprendidas a través de la observación de actitudes violentas previas 
(Domènech e Iñiguez, 2002). También, en el Modelo de coerción (Patterson, 1982) se expone el uso de conductas agresivas en los hijos a partir de la influencia de las pautas educativas inadecuadas utilizadas por los progenitores. A su vez, las Teorías instintivas y psicosociales (Tobeña, 2003) concluyen que la agresividad es necesaria para la supervivencia de la especie y, por lo tanto, no debe frenarse totalmente. Y, por último, en el Modelo ecológico se incorpora una visión holística del fenómeno y lo expone teniendo presente otros aspectos que afectan a la aparición de este tipo de violencia (Cottrell y Monk, 2004).

De esta manera, se observa que son numerosos los factores que influyen en el desarrollo de este tipo de violencia, factores presentes en la sociedad actual caracterizada por el individualismo, el cumplimiento del deseo personal frente al bienestar del otro, la búsqueda de la felicidad individual, el distanciamiento entre los deberes de los hijos y los derechos de los padres, y las diferencias en lo tecnológico entre padres e hijos (Lipovetsky, 2005). Por lo tanto, $\mathrm{y}$ al igual que en otros fenómenos sociales como la violencia de género, el acoso escolar entre otros, se trata de un problema que siempre se encuentra bajo la supervisión de los adultos y que, en cierto modo, ha pasado del ámbito privado al público (López, 2017).

Como consecuencia, cada vez más, desde diferentes esferas públicas tales como el ámbito legislativo, el ámbito profesional y los medios de comunicación, empieza a emerger un discurso propio y a la vez de responsabilidad compartida ante la violencia filioparental. La legislación existente está íntimamente relacionada con el ámbito profesional, en lo que se refiere tanto a la intervención con las familias como a la protección de los agresores menores y de las víctimas. Los profesionales que abordan este tipo de problemática, a su vez, deben conocer la normativa vigente existente al respecto. Los medios de comunicación, por su lado, ayudan a poner en contacto a todos los agentes que intervienen en esta problemática social. De hecho, el incremento de casos de violencia de género y familiar que muestran los medios de comunicación ha desencadenado que las familias se sitúen en el punto de mira (Somovilla, Aguilera, Berrocal y Domínguez, 2016). De este modo, los medios de comunicación pueden ser una vía tanto para la acentuación de los problemas que generan los contenidos violentos como para su prevención y educación ante los mismos (Barrios, 2005).

En este sentido, se hace necesario explorar el discurso social sobre la violencia filioparental en los medios de comunicación, dado el alcance y proyección social que tienen en la sociedad en la que vivimos. Como consecuencia, la sociedad de la información, para las ciencias sociales, ofrece una amplia modalidad de medios de expresión audiovisual que permite al fenómeno de la violencia filioparental hacerse accesible para la opinión pública, a través de noticias, reportajes periodísticos, documentales, foros y webs (Domínguez y Montalbán, 2014). De hecho, las producciones audiovisuales que tratan de asuntos relacionados con este tipo de violencia reflejan cómo negocian los participantes significados comunes con respecto a las causas, factores y consecuencias del origen de los actos violentos, partiendo de sus experiencias y contextos culturales particulares (Bautista, 2009).

Por lo tanto, en la presente investigación se persigue conocer cómo se construye socialmente la violencia filioparental en los medios de comunicación, especialmente en diferentes producciones audiovisuales que persiguen la visibilidad sobre este fenómeno. Desde una perspectiva social, este tipo de materiales se consideran como un "espejo privilegiado" peculiar y muy significativo de la realidad social que generalmente presentan fines didácticos y de difusión social (Loscertales y Nuñez, 2008). Específicamente, se quiso analizar qué estrategias discursivas se usan para legitimar y construir este hecho o, por el contrario, para deslegitimarlo y, de la misma forma, conocer las opiniones de los distintos agentes implicados sobre la violencia filioparental en estos medios.

\section{Material y método}

\subsection{Materiales}

En la presente investigación se realizó un análisis cualitativo discursivo del contenido textual de diferentes documentales sobre la violencia filioparental. Se empleó como criterio lógico intencional para la búsqueda de la muestra el hecho de que se tratara de documentales sobre la violencia filioparental de carácter nacional, producidos por distintas cadenas de televisión y que incluyeran las perspectivas de los dife- 
rentes agentes implicados (profesional, víctima y agresor). Se seleccionaron nueve documentales de treinta y dos visionados:

\section{Para todos la 2: debate la violencia filiopa-} rental, centra su interés en las declaraciones y opiniones de expertos y profesionales en este tipo de problemática. Principalmente, se muestra una descripción generalista del perfil de los agresores y víctimas. También, se tratan los factores que influyen en el mantenimiento de este tipo de violencia, las diferentes formas de intervención, así como las nuevas propuestas o líneas de actuación al respecto.

2. Padres maltratados, expone las vivencias tanto de las víctimas como de los perpetradores. Además, presenta la experiencia de diversos profesionales y expertos del ámbito social y jurídico. El tema central del documental es la "soledad y el sentimiento de abandono y de estigmatización" que muestran los padres para afrontar el problema.

3. ¿Por qué agreden los hijos a los padres?, presenta un debate entre diferentes profesionales que intervienen en estos casos, mostrando cada uno de ellos su experiencia. Se aportan datos que ayudan a conocer en profundidad este fenómeno. Y, se muestran las preocupaciones de la sociedad en general ante este tipo de sucesos.

4. Mi hijo nos pega: testimonio de padres agredidos, describe la realidad de una madre agredida por su hijo y la forma de actuar de un terapeuta experto. Se analiza los factores determinantes en estos casos desde diferentes perspectivas, entre las que destaca: las familiares, educativas y sociales. Se trata de acuñar un responsable a este tipo de actos violentos y se plantea la necesidad de trabajar en la detección, prevención e intervención de dicho problema social.

5. ¿Qué le pasa a mi hijo?, analiza la dramática realidad que viven miles de padres españoles que se ven obligados a denunciar a sus hijos por sufrir maltrato físico o psíquico. Se analiza este nuevo fenómeno social, sus posibles causas y tratamiento en instituciones.

6. Pequeños tiranos, explica la diferencia entre niños tiranos y niños desobedientes. También, cómo reconocer a estos pequeños de "ordeno y mando" y cómo educarlos para que no se conviertan en incorregibles.

7. Educar educándose, trata de que la educación a los hijos es un "laberinto" complejo y necesita muchos prismas, que bien entendidos, no tienen que ser excluyentes.

8. Violencia filioparental, plantea un debate sobre los hijos que maltratan a sus padres. Los expertos, Javier Urra y Josu Arrospide, esclarecen algunos datos y factores influyentes en la violencia filioparental.

9. Los niños tiranos, describe el perfil más común de los denominados niños tiranos, así como las causas y consecuencias de las conductas de estos menores.

En el Cuadro 1 se detalla las características principales del material audiovisual utilizado.

Cuadro 1. Material audiovisual empleado

\begin{tabular}{lcccc}
\hline \multicolumn{1}{c}{ Título } & $\begin{array}{c}\text { Acrónimo } \\
\text { Análisis }\end{array}$ & Duración & Realización & Año \\
\hline $\begin{array}{l}\text { Para todos la 2: debate la violen- } \\
\text { cia filioparental }\end{array}$ & PT2 & $15^{\prime} 24^{\prime \prime}$ & RTVE & 2014 \\
$\begin{array}{l}\text { Padres maltratados } \\
\begin{array}{l}\text { ¿Por qué agreden los hijos a los } \\
\text { padres? }\end{array}\end{array}$ & PM & $51^{\prime} 17^{\prime \prime}$ & Documentos TV & 2012 \\
$\begin{array}{l}\text { Mi hijo nos pega: } \text { testimonio de pa- } \\
\text { dres agredidos }\end{array}$ & MHNP & $25^{\prime} 52^{\prime \prime}$ & ETB2 & 2014 \\
\end{tabular}




\begin{tabular}{lcccc}
\hline \multicolumn{1}{c}{ Título } & $\begin{array}{c}\text { Acrónimo } \\
\text { Análisis }\end{array}$ & Duración & Realización & Año \\
\hline ¿Qué le pasa a mi hijo? & QPMH & $55^{\prime} 20^{\prime \prime}$ & $\begin{array}{c}\text { Pandorga } \\
\text { comunicación }\end{array}$ & 2008 \\
Pequeños tiranos & PT & $10^{\prime} 17^{\prime \prime}$ & Telemadrid & 2010 \\
Educar educándose & EE & $35^{\prime} 44^{\prime \prime}$ & $\begin{array}{c}\text { Ecocentro TV } \\
\text { Programa }\end{array}$ & 2015 \\
Violencia filioparental & VFP & $68^{\prime} 12^{\prime \prime}$ & $\begin{array}{c}\text { sin ir más lejos } \\
\text { Asociación }\end{array}$ & 2013 \\
Los niños tiranos & NT & $34^{\prime} 12^{\prime \prime}$ & $\begin{array}{c}\text { Probienestar familiar } \\
2013\end{array}$ \\
\hline
\end{tabular}

Fuente: Elaboración propia

Estos materiales se localizaron mediante el rastreo de canales diversos, materiales disponibles en asociaciones e instituciones, consulta de repositorios mediáticos de televisiones nacionales y autonómicas, y de universidades. Asimismo, los criterios para su selección fueron el haber recibido mayor difusión durante el periodo comprendido desde el año 2008 hasta la actualidad; presentar mayor diversidad de temas, respecto a las causas, factores y consecuencias de este tipo de violencia; y describir la protección de estas familias tanto en el marco legal y social, en relación a la tipología familiar más frecuente, los roles que desempeñan cada uno de los implicados dentro del ámbito familiar y el modo de intervención en este tipo de casos. Respecto a los criterios de heterogeneidad, se pretendió que fuesen diversos en función del organismo o asociación que lo produjese y que recogieran las vivencias y perspectivas de los agentes involucrados en sus diferentes modalidades, así como las diferentes formas de actuación profesional.

\subsection{Método}

Se propone un diseño cualitativo de corte fenomenológico que permite conocer el significado específico que adquieren los procesos y fenómenos en sus contextos sociales y culturales de emergencia. Este tipo de diseño es heredero del concepto de muestreo teórico o con un propósito desarrollado en el contexto de la Teoría fundamentada (Glaser y Strauss, 1967). Así, se aporta una comprensión más profunda de la violencia filio-parental en los medios audiovisuales analizados. En general, el material de trabajo que se ha empleado no solo lo constituyen las revisiones documentales, sino también las producciones de trabajo o discusión cuya transcripción literal genera un texto de análisis dentro de un escenario social determinado (Cubells, Calsamiglia y Albertín, 2010). Como consecuencia, se han analizado los materiales con un enfoque socioconstruccionista, con especial interés en detectar cuáles son las temáticas que los documentales utilizan para explicar y describir los significados socioculturales relacionados con la violencia filioparental. Concretamente, nos centramos en el análisis del discurso (AD) de la propuesta de Potter y Wetherell (1987) y fundamentalmente recurrimos a la herramienta analítica de los repertorios interpretativos (RI). Los repertorios interpretativos recogen distintos elementos relacionados que el hablante utiliza para construir versiones de eventos, acciones, procesos cognitivos, y otros fenómenos variados. Por ello, más que analizar y contrastar los contenidos o posiciones de diferentes grupos o categorías sociales, nos interesa identificar los ejes reticulares (repertorios) que vehiculizan la producción discursiva a través de lógicas alternativas. La existencia de uno u otro repertorio se reconoce por el tipo de narraciones que lo caracterizan, encontrando en las intervenciones transcritas fragmentos, párrafos, citas o ideas predominantes en alguno de los repertorios y no tanto en el resto (Domínguez y Montalbán, 2017).

\subsection{Procedimiento de análisis}

En el estudio el procedimiento de análisis constó de cuatro fases principales: 
- Búsqueda, selección y preparación del material de análisis (transcripción literal de documentos). El material utilizado en el análisis fueron los textos transcritos resultantes de las producciones audiovisuales. Familiarización con el material mediante lecturas sucesivas, confrontación de mensajes y seguimiento de líneas argumentales, independientemente por parte de cada investigador.

- Identificación de las estrategias discursivas presentes en el material. Las estrategias discursivas son aquellas líneas argumentales comunes que los hablantes utilizan para describir, explicar y, por lo tanto, construir su propia realidad del fenómeno estudiado. El conjunto de estas estrategias discursivas conformaría los distintos repertorios. En general, los repertorios son los marcos discursivos, sustentados por las estrategias discursivas, en los que los participantes fundamentan la retórica de su decir (Domínguez y Montalbán, 2014). Para facilitar la labor de análisis, en esta etapa, se aplicó la versión 7.0 del software Atlas.ti (7.0). El proceso se ha dividido en dos fases diferenciadas, no necesariamente secuenciales, el nivel textual y el nivel conceptual. El programa se empleó en una primera fase (nivel textual) para agilizar la identificación de segmentos de texto (citas) en la construcción de códigos. Posteriormente, dentro de la fase de análisis conceptual, se utilizó para facilitar el establecimiento de relaciones entre códigos para identificar los repertorios.

- Validación y puesta en común por parte de los investigadores de similitudes y divergencias. Así como una revisión de los RI por parte de investigadores externos expertos en dicha temática y en este tipo de análisis (dos nacionales y uno internacional). Finalmente, la obtención de resultados ha estado sometida a la triangulación entre investigadores para garantizar la credibilidad del mismo.

\section{Resultados}

En la construcción discursiva presente en los documentales analizados se han identificado cuatro repertorios interpretativos interrelacionados: Construyendo el perfil de la violencia, Amparo del silencio, Patología del amor y Un síntoma de responsabilidad. En este apartado se ilustra la puesta en práctica de cada repertorio, la preeminencia y el anudamiento que se producen entre ellos, así como los efectos que genera en la propia construcción y presentación del discurso sobre el hecho estudiado.

El primer repertorio, "Construyendo el perfil de la violencia", constituye el soporte principal de la mayoría de los documentales. Se incide en la importancia de ofrecer una descripción del fenómeno atendiendo a las posibles causas y factores, tanto sociales como familiares que lo desencadena. El repertorio "Amparo del silencio" está caracterizado, principalmente, por el predominio de referencias discursivas dirigidas a presentar la violencia filioparental como un hecho enmascarado y mimético. Así, se plantean las causas y consecuencias de mantener este tipo de violencia de manera oculta. A través del repertorio "Patología del amor" se ilustra una relación contradictoria, en cuanto a emociones y sentimientos, entre la víctima y el perpetrador. En el repertorio "Un síntoma de responsabilidad" se manifiesta la importancia que tiene la sociedad en el desarrollo y mantenimiento de estos casos. Se resalta cómo la pérdida de valores tradicionales influye en la evolución social de la familia y en el surgimiento de nuevas prácticas violentas entre ellos. También, se hace referencia a la tendencia que existe de culpabilizar a la víctima e invisibilizar este tipo de violencia.

A continuación, localizamos los repertorios y algunos de sus efectos primordiales sobre la construcción del fenómeno estudiado, a través de la identificación de extractos significativos.

\subsection{Construyendo el perfil de la violencia}

Este repertorio constituye el esqueleto fundamental de la mayoría de intervenciones y se centra en enumerar las causas y posibles consecuencias de la violencia filioparental. Asimismo, se apuesta por dar una descripción generalizada de las víctimas y agresores implicados en esta violencia. Algunas líneas argumentales que sustentan este repertorio se muestran en los siguientes extractos:

Extracto 1 (PQAHP): Los chicos y las chicas agreden por igual, aunque ellos prefieren la violencia física y ellas la psicológica. 
Extracto 2 (VFP): En un 60 o 70\% de los casos es habitual el consumo de tóxicos...Su rendimiento académico es inferior a lo esperado y gestionan erróneamente tanto el tiempo libre como el dinero del que disponen y rehúsan cualquier tipo de responsabilidad u obligación.

En la mayoría de los casos llama la atención la necesidad de generar definiciones acerca de este hecho que dependa exclusivamente del establecimiento de catálogos descriptivos de supuestos indicadores objetivos de riesgos, como los que se presentan en los siguientes extractos.

Extracto 3 (PQAHP): [...] violencia de los hijos hacia los padres es el conjunto de conductas reiteradas de agresiones físicas, como golpes y empujones, verbales (insultos repetidos y amenazas) o no verbales (gestos amenazadores y ruptura de objetos apreciados).

Extracto 4 (QPMH): Hoy lo ha vuelto hacer, es la tercera vez y estoy muy asustada. Ha cogido un cuchillo y me ha amenazado. Acaban de cambiarle la medicación, pero yo no sé a quién creer.

De esta forma, en el tratamiento de la violencia filio-parental como problema social adolece una compleja definición multicausal, derivada principalmente de la construcción del fenómeno como un hecho causante de un trastorno mental, consumo de drogas, estilos educativos permisivos, etc.

Extracto 5 (PT2): "Hay mucho chaval que tiene trastorno, está fastidiao. Y hay mucho chaval que se está volviendo fastidiao por el inicio de edades tempranas de sustancias.

En la mayoría de los documentales se coincide en que las posibles "patologías" existentes en el agresor podrían ser "fruto" de un exceso de derechos del menor ligado a una pérdida de obligaciones como consecuencia de una falta de límites y control de los progenitores.

Extracto 6 (NT): Todo esto es consecuencia de no haber puesto límites a tiempo. ¿Por qué los padres no ponen límites? Hay muchos padres que dicen es que yo no quiero que mi hijo tenga o que pase las mismas privaciones que he pasado yo cuando era pequeño.

Extracto 7 (QPMH): Mis padres autoridad la mínima. Yo llegaba a mi casa y el horario me lo ponía yo, comía cuando yo quería... Yo siempre he hecho lo que he querido.

Este repertorio muestra la crudeza de la realidad de la violencia filioparental, describiendo los perfiles de víctimas y agresores y sus estilos de relación. Refleja cómo los cambios producidos en el ámbito familiar, tales como la incorporación de la mujer al mercado laboral, las nuevas formas de familia, la falta de tiempo para educar por parte de los progenitores y la sobreprotección de los menores, están orientando hacia estilos educativos más permisivos que se muestran como posibles factores causales de este tipo de violencia.

Extracto 8 (VFP):[ ...] hablamos de estilos educativos, familias sobreprotectoras, aquellas que cuando el chico tiene un problema en la escuela, primero escuchan al chico y después al maestro. Las delegativas, esas que consideran que todo el mundo tiene que educar a sus hijos menos ellos, la escuela, sociedad, el maestro, el alcalde $[\ldots]$.

En general, el afán protector hacia los menores y como consecuencia la falta de control hacia estos es tal, que el adolescente adquiere el poder de sus padres y les infunde miedo de forma intencionada, produciéndose una inversión de los roles dentro de la dinámica familiar.

Extracto 9 (PT2): Muchos hijos se sitúan no en verticalidad con respecto a sus padres, sino en horizontalidad.

\subsection{Amparo del silencio}

Este repertorio se caracteriza por el predominio de referencias discursivas a varias cuestiones principales, como son el enmascaramiento de la situación, la ruptura del silencio a través de la denuncia y la normalización de la violencia. En general, se señala la necesidad que tienen las víctimas de mantener oculta las agresiones, relegando tales actos al ámbito privado de la unidad familiar. De este modo, se muestra la violencia como un hecho mimético, en el que cobra especial atención el juego de sentimientos y emociones que se cruzan en el binomio agresor-víctima (relacionado con el siguiente repertorio) y la excesiva sobreprotección del menor (relacionado con el anterior repertorio). 
Extracto 10 (PT): Lo que pasa es que los padres somos muy protectores y eso casi nunca llegas a hacerlo o lo ves como imposible tener que denunciar a un hijo.

Extracto 11 (MHNP): Obviamente para las familias supone una vergüenza de cara a la sociedad, cara a su círculo cercano, exponer que tienen una situación como esta...y sobre todo cómo plantearlo en la sociedad en la que vivimos.

Extracto 12 (PT2): La primera cosa es, no callar, porque cuando esto es un secreto de familia, es mucho más complejo. Por lo tanto, cuando un agresor tiene el amparo del silencio, la agresión va a continuar.

El hecho de negar y normalizar las distintas situaciones violentas por parte de los progenitores dentro del ámbito familiar y social tiene como consecuencia el mantenimiento de los actos violentos. En la mayoría de los documentales se presenta la "denuncia" como la ruptura del silencio $\mathrm{y}$, por lo tanto, como un acto de visibilidad de la situación dada. Se detalla el coste que deben pagar los padres y madres agredidos cuando manifiestan mediante la denuncia la existencia de violencia en su ámbito familiar.

Extracto 13 (PT): Vienen los padres muy desesperados y con un sentimiento de culpabilidad de que no saben lo que han hecho, porque les pasa esto. La judicialización no es buena pero la única opción que les queda es la denuncia.

Extracto 14 (VFP): La mayoría no denuncia por el miedo al qué dirán y porque para el padre o la madre denunciar al hijo es un fracaso absoluto.

Sin embargo, se muestra el hecho de denunciar como la única alternativa viable para solventar estos casos, considerándolo un instrumento terapéutico más que un dato informante de un posible suceso.

Extracto 15 (EE): Cuando nosotros hablamos de la denuncia, no estamos hablando de una forma de criminalizar el caso, no, no, estamos hablando de un instrumento terapéutico que nos puede dar un principio de realidad.

Extracto 16 (PM): La denuncia es la única opción que dan. A mí no me parece el mejor camino, pero sí que hay que hacerlo. Hay que hacerlo porque es la única forma de que presten ayuda.

\subsection{Patología del amor}

Este tercer repertorio destaca la importancia de la institución familiar en la crianza y educación de los hijos. El afecto y el apoyo emocional, especialmente a los hijos, se convierten en los elementos constitutivos de cualquier unidad familiar. En ocasiones, en el caso de la violencia filioparental se producen emociones y sentimientos polarizados (amor-odio) que muchas veces se resuelven con el uso de insultos y amenazas.

Extracto 17 (QPMH): Las agresiones, los insultos es que eran constante, diario.

Extracto 18 (PT2): Sí, quieren querer, pero hay rencor, hay odio, hay malos entendidos, hay subjetividades.

En general y según se explica en los documentales analizados, las relaciones de violencia que se establecen entre los padres y los hijos generan un conflicto emocional en el que prima el dolor, pero en el que de forma habitual permanece el sentimiento de amor. Un amor que se considera patológico y que marca las relaciones en todo el sistema familiar

Extracto 19 (MHNP): Los padres sufren mucho, se esfuerzan mucho por querer a sus hijos, y los hijos sufren mucho cuando agreden a los padres. Alguien lo definió muy bien como una patología del amor.

Extracto 20 (PT): Esos hijos quieren a los padres eso es seguro, sin duda. Y esos padres quieren a sus hijos. Ahora bien, no tienen herramientas no saben cómo comunicarse.

Extracto 21 (VFP): Tengo 87 chicos en la residencia que echan de menos a sus padres, quieren verlos y quieren trasmitirles que lo quieren. Esos padres cuando trabajamos con ello exactamente igual y cuando se juntan son como nitroglicerina son como dos imanes que se atraen pero que lo hemos girado y se repelen.

Igualmente, cobran relevancia las referencias discursivas dirigidas a relacionar este fenómeno de amor patológico con el ciclo de amor presente en la violencia de género.

Extracto 22 (PM): Muchas veces nos comparan con las mujeres maltratadas. No es lo 
mismo. Yo no quiero perder la relación con mi hijo.

\subsection{Un síntoma de responsabilidad}

Este repertorio clarifica la indudable importancia que tiene la sociedad en el desarrollo y en el mantenimiento de estos casos. Recoge el compromiso y responsabilidad de los diferentes actores sociales implicados en la violencia filioparental.

Extracto 23 (PM): Estamos ante menores que yo creo que no han tenido límites, ausencia de límites y desde luego, en los que veo que falla la familia, falla la escuela y falla nuestra sociedad.

Extracto 24 (EE): [...] tiene que ver con la sociedad, o sea no solo en las familias, la sociedad, una cultura más permisiva.

Extracto 25 (PQAHP): No hay que cargar toda la responsabilidad en las familias. También el centro escolar es un centro muy importante donde hay que enseñar a convivir, ....se están haciendo cosas que realmente es un trabajo conjunto, lo que pasa que quien más sufre es en este caso la familia, ese padre, esa madre y ese chaval, y los hermanos, pero es un problema del conjunto de la sociedad.

Las intervenciones se dirigen a mostrar que la responsabilidad de estas situaciones debe ser compartida por todas las esferas públicas que intervienen en este fenómeno. De esta forma, se destaca la importancia del compromiso desde los centros escolares, desde los profesionales, desde los medios de comunicación, etc. así como la urgencia de educar a los adolescentes en el buen uso y consumo de las tecnologías, y de ampliar los recursos sociales ante las necesidades que, en ocasiones, las familias ven descubiertas.

Extracto 26 (EE): Los padres tienen su parte de responsabilidad, por supuesto que sí. Yo en mi caso no intento eludirla en ningún momento, pero también entiendo, que el colegio tiene una responsabilidad; luego vivimos en una sociedad, que hay unos medios de comunicación y hay una sociedad que está ahí".

Extracto 27 (PT2): Estamos en una sociedad que enaltece la violencia, que la protege, que, que la tolera, y que incluso la ama, y por lo tanto podemos ver en estos momentos cómo por ejemplo algunos de los videojuegos más utilizados o más famosos son extremadamente violentos.

Extracto 28 (PT2): [...] efectivamente la violencia está muy normalizada en videojuegos, películas $[\ldots]$.

Extracto 29 (PM): En nuestra asociación de niños con trastornos por déficit de atención e hiperactividad escuchamos a 200 o 300 familias cada día, que van a pedir ayuda porque en aquellos sitios donde la responsabilidad social, gubernamental, institucional, educativa...donde van pidiendo ayuda para el problema que tienen, todavía no se ha encontrado solución.

El hecho de trabajar sobre la recuperación de valores se plantea como posible línea de intervención dirigida a desaprender las conductas aprendidas y a construir una nueva realidad social ante este fenómeno. Se ejemplifica en los siguientes extractos:

Extracto 30 (VFP): Tenemos que rearmarnos en valores morales de respeto y educar en sentimientos.

Extracto 31 (EE): [...] es un problema de valores, esto no es un tema legal, es un tema moral, cívico... Es la sociedad del todo nos vale.

Extracto 32 (PT2): [...Al final todos esos comportamientos son conductas aprendidas y lo mejor de todo es que se pueden desaprender, es decir se pueden corregir.

\section{Discusión y conclusiones}

El estudio llevado a cabo ha permitido conocer las estrategias discursivas desde las que se está construyendo el fenómeno de la violencia filioparental en la actualidad a través de los medios de comunicación social. Los repertorios interpretativos identificados en los documentales analizados muestran la realidad compleja de la violencia de los hijos hacia los padres, señalando las experiencias y responsabilidades de cada uno de los actores implicados.

La experiencia que se vive en las situaciones de VFP refleja claramente el ciclo descrito por Aroca, Lorenzo y Miró (2014) en los roles y dinámicas familiares que la sociedad actual tiende a marcar. El repertorio Construyendo el perfil de la violencia presenta a unos padres sobrecargados en su vida cotidiana ante necesidades especiales de sus hijos y sin el tiempo y recursos suficientes para dedicarles a su edu- 
cación. Las relaciones entre los miembros se deteriora fruto de estilos educativos inadecuados y del intercambio de roles en la dinámica familiar, pasando a ser los hijos los que pretenden imponer el poder y control por encima de sus padres (Omer, 2004; Pereira, 2006).

Estos hijos e hijas agresoras muestran un perfil común en cuanto al sexo coincidente con otros estudios previos (Kennair y Mellor, 2007; McCloskey y Lichter, 2003; Vázquez et al., 2019). Además, se presentan ciertas necesidades de atención especial derivadas de problemas con tóxicos, trastornos de salud mental, etc., lo que complejiza de forma potencial las relaciones familiares.

El ciclo de violencia permanece porque la VFP se mantiene oculta en el ámbito privado familiar durante mucho tiempo. En el repertorio Amparo del silencio se constata el riesgo aún presente a la normalización de las conductas violentas tanto por parte de la sociedad como por las familias donde ocurre. En estas, en un primer momento se intenta negar la situación (Suárez, 2012), y solo cuando el círculo parece insostenible y las personas agredidas no encuentran otra alternativa, optan en la mayoría de los casos por pedir ayuda al sistema judicial a través de la interposición de una denuncia. En este sentido, los expertos destacan la función terapéutica de la denuncia como medio para el desbloqueo del proceso y para el inicio de una necesaria intervención socioeducativa con la unidad familiar (López-Molina, 2014, Peligero, 2016). Esta intervención ha de implicar a toda la familia, ya que a pesar de hacerse visible el conflicto a través de la actitud violenta del menor, resulta fundamental para la mejora de las relaciones la participación activa de los padres.

En todo este proceso, los sentimientos $\mathrm{y}$ emociones se revelan contradictorios, tal como se ha evidenciado en el repertorio $\mathrm{Pa}$ tología del amor. Los agresores y agresoras tienen sentimientos positivos hacia sus padres, aunque su comportamiento violento exprese lo contrario, y las víctimas también presentan sentimientos positivos hacia sus hijos, intentando protegerlos en todo momento, ya sea a través del ocultamiento inicial de la situación o de la solicitud de ayuda a distintos organismos e instituciones. En este sentido, se relaciona este fenómeno con el ciclo de amor presente en la violencia de género. De este modo, la violencia filioparental se vincula a la violencia machista ("muchas veces nos comparan con mujeres maltratadas") puesto que, en la mayoría de los casos, son las madres quienes son las maltratadas (Sánchez et al, 2019). La prevalencia de la madre como principal víctima reside en que aún sigue considerándose como la cuidadora principal y responsable de la educación de los hijos. Por tanto, está sujeta a una mayor probabilidad de enfrentamientos con ellos.

Aun cuando existen autores que consideran que la familia es la institución más violenta de la sociedad (Alba, 2006; Aroca, Cánovas y Alba, 2012), es sin duda en ella donde a su vez se encuentran los elementos necesarios para el desarrollo de respuestas saludables ante los problemas, siendo reconocida como la unidad básica de socialización del afecto (Ibabe, 2015).

Por último, y junto a las consecuencias y responsabilidad familiar en la violencia filioparental (Trujillo, Sahagún, Cárdenas y Ramírez, 2016), el repertorio Un síntoma de responsabilidad muestra la tarea corresponsable de todos los actores implicados en su aparición y desarrollo, evidenciando la urgencia de su abordaje coordinado y global (Lipovetsky, 2005). Es necesario desaprender para aprender, contribuyendo desde todas las esferas y medios posibles al desarrollo de mejores relaciones personales, familiares y sociales. En esta tarea y de acuerdo con Barrios (2005), la función preventiva y educativa de los medios de comunicación social es una vía de especial utilidad. Asimismo, se recomienda actuar de manera integral en el núcleo familiar. De este modo, se responsabiliza del problema a todos los miembros de la familia. Como señala Claver (2017), la violencia es un fenómeno aprendido que se tiene que sustituir por otro que consiga iguales resultados individuales sin desmembrar y destruir el sistema familiar.

Entre las limitaciones que presenta esta investigación destacan la ausencia de personas entrevistadas e implicadas en este proceso. Se ha considerado que los medios de comunicación son claves para el estudio de este tipo de fenómenos. A través, de estos se aprenden y se visionan determinadas conductas sobre las que son necesarias indagar para desarrollar nuevos programas de prevención e intervención. De este modo, es necesario centrar las investigaciones en estos comportamientos tales como: la presencia de violencia psicológica en las mujeres, la invisibilización de este tipo de maltrato, la violencia filioparental centrada en las 
madres, entre otros. También, se recomendable incorporar más trabajos cualitativos, o que combinen el enfoque cualitativo con el cuantitativo, con la finalidad de contar con diferentes aproximaciones y tener mayor información acerca del fenómeno.

En resumen, este estudio muestra la representación social y mediática de la violencia filioparental a través de los medios de comunicación social. En consecuencia, se contribuye a una doble visibilización de esta realidad social. Por un lado, al desvelar las experiencias y sentimientos presentes en las familias que viven esta situación, y por otro, al demandar el compromiso y responsabilidad de todas las esferas sociales implicadas en su prevención y atención. En este sentido, este trabajo se presenta como invitación a seguir reflexionando sobre este fenómeno y sobre sus implicaciones para la intervención sociofamiliar.

\section{Referencias bibliográficas}

Alba, J.L. (2006). Fundamentos de psicología jurídica e investigación criminal. Salamanca: Ediciones universitarias.

Aroca, C., Cánovas P. y Alba J.L. (2012). Características de las familias que sufren violencia filio-parental: un estudio de revisión. Educatio Siglo XXI, 30(2), 231-254.

Aroca, C., Lorenzo, M. y Miró, C. (2014). La violencia filioparental: un análisis de sus claves. Anales de Psicología, 30 (1), 157-170. doi: 10.6018/analesps.30.1.149521

Asociación Probienestar familiar (2013). Los niños tiranos [CD].

Bandura, A. (1971). Social learning theory. New York: General learning press.

Barrios, C. (2005). La violencia audiovisual y sus efectos evolutivos: un estudio teórico y empírico. Сomunicar, 25. doi: 10.3916/C25-2005-074

Bautista, A. (2009). Relaciones interculturales en educación mediada por narraciones audiovisuales. Comunicar, 17, 149-156. doi:10.3916/c33-2009-03-006

Calvete, E., Orue, I. y González-Cabrera, J. (2017). Violencia filio parental: comparando lo que informan los adolescentes y sus progenitores. Revista de psicología clínica con niños y adolescentes, 4 (1), 9-15.

Castilla, R. y Palma, M.O. (2014), "El maltrato a personas mayores en el ámbito familiar. Aproximación a la situación en Málaga (España)". Trabajo Social Global. Revista de Investigaciones en Intervención Social, 4 (7), 20-35.

Claver, E. (2017). Aproximación teórica a la violencia filioparental. Redes,35,21-32.

Cottrell, B. y Monk, P. (2004). Adolescent-to-Parent Abuse. A Qualitative Overview of Common Themes. Journal of Family Issues, 25 (8), 1072-1095. doi: 10.1177/0192513X03261330

Cubells, J., Calsamiglia, A. y Albertín, P. (2010). El ejercicio profesional en el abordaje de la violencia de género en el ámbito jurídico-penal: un análisis psicosocial. Revista Anales de Psicología, 26(2), 369377.

Documentos TV. (2012). Padres maltratados [vídeo]. Disponible en: https://www.documentales-online. com/tag/documentos-tv/

Domènech, M. e Íñiguez, L. (2002). La Construcción Social de la Violencia. Athenea digital, 2, 1-10.

Domínguez, L. y Montalbán, F.M. (2014). Estrategias discursivas de normalización en la producción audiovisual sobre la homoparentalidad. Revista Arbor, 190 (769), a166. doi: 10.3989/arbor.2014.769n5005

Domínguez, L. y Montalbán, F.M (2017). Alternativas en el debate sobre el matrimonio igualitario en Europa y América Latina. Andamios, 14, 335-357.

ETB2. (2014). ¿Por qué los hijos agreden a los padres? [vídeo]. Disponible en: https://www.youtube.com/ watch? $=$ ehLb4z1itDc

ETB2. (2015). Mi hijo nos pega: Testimonios de padres agredidos [vídeo]. Disponible en: https://www.eitb. eus/es/television/programas/sin-ir-mas-lejos/reportajes-adela-ucar/videos/detalle/3079318/video-testimonios-padres-agredidos-sus-hijos--adela-ucar/

Glaser, B. \& Strauss, A. (1967). The discovery of Grounded Theory: Strategies for qualitative research. Chicago: Aldine.

Gómez, A., Gala, F.J., Lupiani, M., Bernalte, A., Miret, MT., Lupiani, S. y Barreto, MC. (2007). El “bullying" y otras formas de violencia adolescente. Cuadernos de Medicina Forense, 13, 165-177.

Ibabe, I., y Jaureguizar, J. (2011). ¿Hasta qué punto la violencia filio-parental es bidireccional? Anales de psicología, 27 (2), 265-277. 
Ibabe, I. (2015). Predictores familiares de la violencia filio-parental: el papel de la disciplina familiar. Anales de psicología, 31(2), 615-625. doi:10.6018/analesps.31.2.174701

Kennair, N. \& Mellor, D. (2007). Parent Abuse: A Review. Child Psychiatry and Human Development, 38 (3), 203-219. doi: 10.1007/s10578-007-0061-x

Lipovetsky, G. (2005). El crepúsculo del deber: La ética indolora de los nuevos tiempos democráticos. Barcelona: Anagrama.

López, M.G. (2017). Influencia del clima escolar y familiar en el acoso escolar y cibernético de universitarios. Revista Mexicana de Investigación en Psicología, 9(1), 31-44.

López-Molina, C. (2014). Una aproximación a los conflictos en el ámbito familiar y doméstico en familias de menores con medidas judiciales en medio abierto, en la sociedad actual. Revista internacional de Trabajo Social y Bienestar Azarbe, 3, 259-262.

Loscertales, F. y Nuñez, T. (2008). Ver cine en TV: una ventana a la socialización familiar. Comunicar, 16, 137-143. doi: 10.3916/c31-2008-01-017

McCloskey, L.A. y Lichter, E. (2003). The Contribution of Marital Violence to Adolescent Aggression Across Different Relationships. Journal Interpersonal Violence, 18(4), 390-412. doi: $10.1177 / 0886260503251179$.

Medina, A., Arévalo, A y Durán, A. (2015). Necesidades, expectativas y sueños sobre la relación de pareja en hombres remitidos para atención psicológica por denuncias de violencia intrafamiliar. Universitas Psychologica, 14(1), 205-2018. doi: 10.11144/Javeriana.upsy13-5.nesr

Molina, J.E. y Moreno, J.H. (2015). Percepción de la experiencia doméstica en mujeres víctimas de maltrato de pareja. Universitas Psychologica, 14 (3), 997-1008. doi: 10.11144/Javeriana.upsy14-3.pevd

Omer, H. (2004). Non violent resistance: a new approach to violent and self-destructive children. Cambridge: Cambridge University Press.

Pandorga comunicación. (2008). ¿Qué le pasa a mi hijo? [vídeo]. Disponible en: https://player.vimeo.com/ video/83332911

Patterson, G.R. (1982). A Social Learning Approach: Coercitive Family Process. Oregón: Castalia.

Peligero, A.M. (2016). La violencia filioparental en la violencia familiar. IPSE, 9, 69-84.

Pereira, R. (2006). Violencia filio-parental. Un fenómeno emergente. Revista Mosaico, 36, 8-9.

Potter, J. y Wetherell, M. (1987). Discourse and social psychology: Beyond attitudes and behavior. Londres: Sage.

Programa sin ir más lejos. (2012). Violencia filioparental $[C D]$.

RTVE. (2014, octubre 10). Para todos la 2: debate de violencia filioparental [vídeo]. Disponible en: http:// www.rtve.es/alacarta/videos/para-todos-la-2/para-todos-2-debate-violencia-filioparental/2801656/

Sears, R., Maccoby, E. y Levin, H. (1957). Patterns of child rearing. Row \& Peterson: Illinois.

Steinmetz, S.K. (1978). Battered parents. Society, 15, 54-55.

Somovilla, E., Aguilera, J., Berrocal, R. y Domínguez, L. (2016). Análisis discursivo de la violencia filioparental: ámbito profesional y familiar. Revista de Trabajo Social Glogal-Global Social Work: Revista de investigaciones e intervención social, 6, 96-120. http://hdl.handle.net/10481/41931

Suárez, B. (2012). Violencia filio-parental: Aproximación a un fenómeno emergente. Revista de humanidades y ciencias sociales, $11,73-107$.

Telemadrid. (2010). Pequeños tiranos [vídeo]. Disponible en: https://www.youtube.com/watch?v=nLT8Djweztw

Tobeña, A. (2003). Anatomía de la agresividad humana. Barcelona: Debolsillo.

Trujillo, J., Sahagún, M., Cárdenas, R. y Ramírez, A.F. (2016). Las consecuencias de la violencia filio-parental reflejadas en una historia de vida. Cuadernos de Trabajo Social, 29(1), 119-128. doi:10.5209/ rev_CUTS. 2016.v29.n1.47159

Urra, J. (2015, junio 28). Educar educándose [vídeo]. Disponible en: https://www.youtube.com/watch?v=CeTNxp4mmjg

Vázquez, V., Romo, R.J., Rojas, J.L., Gónzalez, M.P. y Yedra, L. (2019). Violencia filioparental en adultos emergentes mexicanos: Un análisis exploratorio. Revista electrónica de Psicología Iztacala, 22 (3), 2534-2551. 